\title{
Internationale Aspekte der sozialen Sicherheit in den franco- phonen Ländern südlich der Sahara
}

\author{
Von Otto Kaufmann
}

\section{Zur Entwicklung der Systeme sozialer Sicherheit in Afrika}

Während der Kolonialzeit bestanden in den francophonen Ländern Schwarzafrikas ${ }^{1}$ lange Zeit keine klassischen (europäischen) Systeme sozialer Sicherheit. Ein gewisser sozialer Schutz wurde zwar durch einige wenige, von der belgischen oder französischen Kolonialmacht eingeführten Maßnahmen garantiert, ${ }^{2}$ aber die afrikanische Bevölkerung mußte sich hauptsächlich auf die traditionelle Solidarität verlassen. ${ }^{3}$ Im Hiblick auf die Wanderbewegungen in den betreffenden afrikanischen Gebieten brachte der zum Ende der Kolonialzeit begonnene Aufbau von sozialen Sicherungssystemen keine erheblichen Probleme mit sich, denn in allen von derselben Kolonialmacht abhängigen Gebieten bestand im allgemeinen eine einheitliche Gesetzgebung und die Zusammenarbeit der Einrichtungen sozialer Sicherheit innerhalb des Hoheitsbereiches einer Kolonialmacht war gewährleistet; Unterschiede betrafen in aller Regel nur die Höhe der Sozialleistungen. ${ }^{4}$

Die Situation änderte sich nach der Erlangung der Unabhängigkeit der afrikanischen Länder um 1960. Die nun souveränen Staaten schufen entweder neue Systeme sozialer Sicherheit oder ergänzten die von der früheren Kolonialmacht eingeführten. ${ }^{5}$ Die wirtschaftlichen Möglichkeiten und die sozialen Ambitionen der verschiedenen Länder waren die hauptsächliche Ursache für die festzustellenden unterschiedlichen Entwicklungen im Bereich der sozialen Sicherung, die ganz spezifische Schwierigkeiten im Hinblick auf die internationale Mobilität der Arbeitnehmer in Afrika mit sich brachten und noch bringen, vor allem in Anbetracht der Tatsache, daß die traditionelle Solidarität

1 Die Länder sind: Benin, Burundi, Djibouti, Elfenbeinküste, Gabun, Guinea, Kamerun, Kongo, Madagaskar, Mali, Mauretanien, Niger, Obervolta, Ruanda, Senegal, Togo, Tschad, Zaire, Zentralafrikanische Republik.

2 Gonidec, P. F./Kirsch, M., Traité du droit du travail des territoires d'Outre-Mer, Paris LGDJ, 1958, S. $19 \mathrm{ff}$.

3 Elwert, G., Die Elemente der traditionellen Solidarität - Eine Fallstudie in Westafrika, Kölner Zeitschrift für Soziologie und Sozialpsychologie, 1980, S. 641-704. Songuemas, $N$., Les institutions traditionelles et la notion de sécurité sociale dans les sociétés africaines, Cahiers africains de sécurité sociale, no. 3, 1967, S. 19-29.

4 In den belgischen Kolonien wurden erste Systeme 1949 eingeführt, in den französischen zwischen 1955 und 1958.

Dazu: BIT, Les problèmes du travail en Afrique, Etudes et documents, Nouvelle série, no. 48, Genève 1958, S. 425 ff. S. auch Gonidec/Kirsch (Fn. 2), S. 68 ff.

5 Mouton, P., La sécurité sociale, in: Encyclopédie juridique de l'Afrique, Bd. 8, Droit des relations professionelles (travail, sécurité sociale et fonction publique), NEA, Abidjan, Dakar, Lomé, 1982, S. 267 ff. 
dem lohnabhängigen Wanderarbeiter immer weniger nützen kann, da seine Lebensweise von der althergebrachten immer mehr abweicht. Um den Wanderarbeitnehmern einen effektiven sozialen Schutz zu gewährleisten, ist es also notwendig, Maßnahmen in diesem Bereich zu treffen, die über den nationalen Rahmen hinausreichen und diese Bevölkerungsschicht vor Nachteilen bewahrt, die durch die Migration entstehen können. ${ }^{6}$ Es handelt sich dabei um Harmonisierungs- und Koordinierungsmaßnahmen im internationalen Rahmen.

Im folgenden sollen nun einige Ausführungen über den Begriff der Harmonisierung und Koordinierung, sowie über die in den afrikanischen Ländern bestehenden Disparitäten im Bereich der sozialen Sicherheit gemacht werden, durch welche eine Angleichung der verschiedenen Rechtsordnungen geboten erscheint. Anschließend werden die Grundzüge der Harmonisierung und Koordinierung, wie sie in den afrikanischen Ländern gestaltet werden, erläutert.

\section{Harmonisierung und Koordinierung}

Die vorstehend angeführten Probleme können durch Harmonisierungsmaßnahmen, ${ }^{7}$ die im nationalen oder im internationalen Rahmen ansetzen können, abgebaut werden. Für die afrikanischen Länder ist vor allem die Harmonisierung im internationalen Rahmen von Bedeutung, da es gilt, die mit der Wanderarbeit verbundenen Nachteile auszugleichen. Sie kann sowohl das gesamte Sozialrecht erfassen, als auch im Rahmen einzelner sozialrechtlicher Teilsysteme gedacht werden, oder auch nur Begriffsbestimmungen der sozialen Sicherheit betreffen. Das setzt aber den Rechtsvergleich der von Harmonisierungsmaßnahmen betroffenen Rechtssysteme voraus. ${ }^{8}$ Durch eine Harmonisierung werden verschiedene Rechtssysteme oder Teilbereiche davon einander angeglichen. Aufgrund der nationalen Interessen und Besonderheiten ist das aber im allgemeinen nur bis zu einem gewissen Grad möglich. Denn die Harmonisierung ist im weiten Sinne auch ein wirtschaftliches und politisches Integrationsinstrument und setzt für ihre Realisierung die Uberwindung erheblicher technischer und politischer Hindernisse voraus. Eine unverzichtbare Voraussetzung für ihre Durchführung ist auch das Bestehen einer internationalen Solidarität zwischen allen betroffenen Ländern. Bei der Umsetzung dieser politischen Solidarität in die praktische Angleichung der Systeme sozialer Sicherheit kann aber schon der oft recht unterschiedliche Entwicklungsstand in den einzelnen Ländern einen Hemmschuh darstellen, weil einerseits ein erreichter hoher Standard

6 Les migrations internationales de travailleurs africains au sud du Sahara, Revue internationale du travail, no. 3 , 1957, S. 325 ff.

7 Eingehend zum Begriff der Harmonisierung: von Maydell, Harmonisierung von Systemen sozialer Sicherheit - Herrn Prof. Dr. Wertenbruch zum 65. Geburtstag gewidmet. Die Sozialgerichtsbarkeit, Heft 4, 1984, S. 142-145.

8 Zur Sozialrechtsvergleichung: Zacher, H. F. (Hrsg.), Methodische Probleme des Sozialrechtsvergleichs, Bd. 1 der Schriftenreihe für internationales und vergleichendes Sozialrecht, 1977. 
(Organisation, Leistungszugang und Leistungsvoraussetzung) der Sicherungssysteme nicht abgebaut werden sollte, andererseits aber ein verhältnismäßig niederer Standard nicht ohne weiteres wesentlich angehoben werden kann. Eine Harmonisierung, die sich die Integration zum Ziel setzt, ist demnach nur langfristig durchführbar. Darum ist der Zweck der von den afrikanischen Ländern angestrebten Harmonisierung nicht die totale Angleichung und Vereinheitlichung der nationalen Systeme sozialer Sicherheit, sondern eine qualitative Veränderung dieser Systeme mit dem Vorsatz, auf ein gemeinsames Ziel zuzugehen. ${ }^{9}$

Die bei der Durchführung der Harmonisierung zu überwindenden Hindernisse können durch eine Koordinierung, die als Ergänzung und auch als Vorstufe der Harmonisierung angesehen werden kann, teilweise bewältigt werden. Die Koordinierungsinstrumente ermöglichen die Anwendung der von den verschiedenen Systemen sozialer Sicherheit vorgesehenen Regelungen über den nationalen Rahmen hinaus. Aber die nationale Autonomie und Individualität der Rechtsordnungen bleiben vollständig erhalten, da es sich bei der Koordinierung lediglich um ein Ineinandergreifen von Regelungen handelt, das dem nationalen Recht seinen Charakter läßt, aber dem Unterschied und der Verschlossenheit nach außen Grenzen setzt. ${ }^{10}$

Koordinierungsmaßnahmen beugen einem eventuellen Verlust der von einem Arbeitnehmer erworbenen Rechte und Anwartschaften vor, wenn dieser in ein anderes Land übersiedelt. Effektiv wird dieser Schutz durch eine enge Kooperation zwischen den zuständigen nationalen Institutionen sozialer Sicherheit im Rahmen der Koordination. Die Durchführung dieser Maßnahmen begegnet schon innerhalb strukturell mehr oder weniger ähnlicher Systeme, wie sie die francophonen Länder aufweisen, erheblichen Schwierigkeiten. Dennoch sollten auch die anglophonen Länder an den Harmonisierungs- und Koordinierungsmaßnahmen beteiligt werden, zumindest wenn sie mit den francophonen in einer geographischen Zone liegen. So könnten auch erste Schritte in Richtung einer wirklichen afrikanischen Einheit getan werden und die durch die Kolonisierung errichteten sprachlichen, aber vor allem politischen und strukturellen Zonen einander näher gebracht werden. ${ }^{11}$

Harmonisierung und Koordinierung verlangen jeweils unterschiedliche Instrumente für ihre Durchführung, zeigen aber als gemeinsames Merkmal eine "Deterritorialisierung" der Systeme sozialer Sicherheit. Einerseits werden vorübergehende Tätigkeiten im Inland vom nationalen System ausgenommen (Einstrahlung), andererseits werden vorübergehende Tätigkeiten im Ausland vom nationalen System erfaßt (Ausstrahlung).

9 Zu diesem Problem für die EG: Mertens, J., L'harmonisation des régimes de sécurité sociale dans les Communautés Européennes, Revue belge de sécurité sociale, 1982, S. 249 ff.

10 Zacher, H. F., Horizontaler und vertikaler Sozialrechtsvergleich, in: Sozialrechtsvergleich im Bezugrahmen internationalen und supranationalen Rechts, Bd. 2 der Schriftenreihe für internationales und vergleichendes Sozialrecht, 1978, S. 16.

11 Ausführlich zu diesen Problemen: Encyclopédie juridique de l'Afrique (Fn. 5), Bd. 1, L'Etat et le droit; Bd. 2, Droit international et relations internationales. 


\section{Gründe für Harmonisierungs- und Koordinierungsmaßnahmen}

Nicht nur soziale Abwägungen zugunsten der Wanderarbeiter, sondern auch wirtschaftliche, zwingen die betroffenen afrikanischen Staaten, einschlägige Maßnahmen zu ergreifen. Da die Zahl der afrikanischen Wanderarbeiter in nicht unerheblichem Maße zugenommen hat, ${ }^{12}$ stellt die Disparität zwischen den verschiedenen nationalen Systemen einen Störfaktor dar. Die Länder mit den entwickeltsten Systemen sozialer Sicherheit stellen einen Anziehungspol für ausländische Arbeitnehemr dar und werden zu Einwanderungsländern. Die dadurch auf den Unternehmen und vor allem auf den Einrichtungen der sozialen Sicherheit lastenden Kosten können ggf. entwicklungshemmend sein, gerade in den sich in Entwicklung befindenden afrikanischen Ländern, wo die wirtschaftliche Stabilität bei weitem nicht so gesichert ist wie in den Industrieländern. Die Länder mit einem rudimentären System sozialer Sicherheit hingegen verlieren zu viele Arbeitnehmer und müssen dadurch gegebenenfalls eine Schwächung ihrer Wirtschaft in Kauf nehmen, auch wenn die bestehenden Wirtschaftsunternehmen dieser Länder durch die geringen sozialen Kosten einen Wettbewerbsvorteil erzielen können. Harmonisierungsmaßnahmen können diese Verzerrung hinsichtlich der finanziellen Lasten, aber auch hinsichtlich des sozialen Schutzes innerhalb der verschiedenen Systeme abbauen.

Manche Disparitäten zwischen den verschiedenen Systemen gehen auf die Einführung der Schutzsysteme durch die Kolonialmacht zurück. In den ehemaligen französischen Gebieten stellt das von der Kolonialmacht eingeführte Familienleistungssystem immer noch den Eckpfeiler des sozialen Schutzsystems dar. ${ }^{13}$ Die Aufrechterhaltung dieses Familienleistungssystems ist aber wegen der demographischen Expansion sehr kostspielig. Dadurch kann der Auf- und Ausbau anderer Zweige der sozialen Sicherheit gefährdet werden. Dennoch scheint es aus politischen Gründen nicht ohne weiteres möglich, das Familienleistungssystem zu entlasten, indem statt Geldleistungen Sachleistungen erbracht würden, weil sich die leistungsberechtigten Arbeitnehmer einer Änderung massiv widersetzen würden. ${ }^{14}$

Harmonisierungs- und Koordinierungsmaßnahmen im internationalen Rahmen werden zwar durch die schlechte Anpassung der einzelnen Sicherungssysteme an die jeweils vorherrschende nationale Bevölkerungsstruktur nicht beeinträchtigt, jedoch wirft die

121970 wurde die Zahl der Wanderarbeiter in Westafrika auf mehr als 1 Mio. geschätzt (Hance, W. A., Population Migration and Urbanization in Africa, New York, Columbia University Press, 1970). 1973 ergab eine Schätzung über 3 Mio. (BIT, Emplois, Statuts et conditions des travailleurs migrants et des autres travailleurs possédant la nationalité d'autres pays africains, 4eme Conférence régionale africaine de l'OIT, Nairobi, 1973, Rapport II, p. 4).

S. a. Zachariah, K. C./Condé J., Migration in Westafrica: Demographic Aspects, Worldbank 1981, O.U.K. Press Bd., London.

13 Mouton, P., La sécurité sociale en Afrique au sud du Sahara, Internationales Arbeitsamt, Genf 1974. Gonidec/Kirsch (Fn. 2), S. $627 \mathrm{ff}$.

14 Issa-Sayegh, J., Communication: Les problèmes internationaux de la sécurité sociale an Afrique Occidentale; Colloque sur l'intégration en Afrique de l'Ouest, Dakar 28 mars-2 avril 1978, Université de Dakar, S. 4. 
Defacto-Beschränkung des sozialen Schutzes auf die städtische Arbeitnehmerschaft erhebliche Probleme auf. ${ }^{15}$ Insbesondere wird dadurch die Landflucht begünstigt und die Ubervölkerung der urbanen Zentren bewirkt, was dann Auswirkungen auf das Funktionieren der Schutzsysteme zeitigt. Die derzeit in den meisten afrikanischen Ländern bestehende Struktur der Sicherungssysteme sollte daher an die veränderte Sozialstruktur angepaßt werden, ${ }^{16}$ vor allem in Anbetracht der Tatsache, daß die Situation vieler ländlicher Arbeitnehmer mit der der urbanen lohnabhängig Beschäftigten verglichen werden kann. ${ }^{17}$

Erschwerend für die internationale Zusammenarbeit kommen noch die z. T. gravierenden Defizienzen in für die sozialen Sicherungssysteme komplementären Dienstleistungsund Verwaltungsbereichen jeglicher Art hinzu. ${ }^{18}$ Defizienzen, insbesondere im personellen Bereich innerhalb der Institutionen sozialer Sicherheit mögen wohl der Grund sein, daß manche Staaten bestimmte Zweige der sozialen Sicherheit privaten Organismen anvertrauen und auf eine öffentliche Verwaltung verzichten. Davon können Angleichungsmaßnahmen und in noch verstärktem Maße Koordinierungsmaßnahmen zwischen den verschiedenen Rechtsordnungen direkt betroffen sein.

Hinsichtlich der aktuellen Unterschiede zwischen den Systemen sozialer Sicherheit in den einzelnen Ländern kann festgestellt werden, daß neben den schon angeführten Familienleistungen alle Systeme Mutterschaftsleistungen erbringen und als Ergänzung dazu Gesundheits-, Sozial- und Familienmaßnahmen der Kassen vorsehen. ${ }^{19}$ Der Schutz gegen das Risiko Krankheit ist hingegen in den meisten Ländern nur sehr dürftig entwikkelt und oft den Arbeitgebern überlassen. ${ }^{20}$ Das gilt sowohl für die Einkommensersatzleistungen als auch für die Dienst- und Sachleistungen im Krankheitsfall. Zweifelsohne entsteht dadurch innerhalb der einzelnen Länder ein Gefälle hinsichtlich des sozialen Schutzes zwischen den großen, finanzkräftigen Unternehmen einerseits, und den kleinen, finanzschwächeren, andererseits. Ausnahmen bilden Guinea und Senegal, wo alle Arbeitnehmer einer Pflichtversicherung für das Risiko Krankheit unterliegen. ${ }^{21}$ Mali hat ein Mischsystem eingeführt, das sich durch eine Verbindung von der betrieblichen Versorgung im Krankheitsfall mit einer Krankenversicherung auszeichnet. Ähnlich ist die Situation in Mauretanien, wo die Sozialversicherungskasse den Krankenhäusern für die

15 Dazu: Mouvagha-Tchioba, E. G., Les principes du système de sécurité sociale français sont-ils applicables à l'Afrique Noire francophone? Institut international d'études sociales, Genève, 1979.

16 Cockburn, Ch., Die Rolle der sozialen Sicherheit in der Entwicklung, in: Internationale Revue für soziale Sicherheit, 1980, S. 378. Improvement and Harmonisation for Social Security Systems in Africa, 5th African Regional Conference, Report II, Abidjan 1977, ILO, Genf, S. 40 f.

17 Issa-Sayegh, J. (Fn. 14), S. 5.

18 Improvement and Harmonisation of Social Security Systems in Africa (Fn. 16), S. 30 f.

19 Dazu: Diabata, D., L'action sanitaire et sociale des institutions de sécurité sociale des pays francophones d'Afrique, in: IVSS, Documentation de sécurité sociale, série africaine, no. 6, Genf 1983, S. 7 ff.

20 Wenn die bestehenden Tarifverträge keine entsprechenden Bestimmungen enthalten, ergeben sich die Pflichten der Arbeitgeber aus den 1955 erlassenen Vorschriften über die ärztliche Versorgung in den Unternehmen (Arrêtés généraux nos. 395, 397, 398, JOAOF, 29 janvier 1955).

21 Zum System sozialer Sicherheit im Senegal, s. Kaufmann, O., Le droit de la sécurité sociale au Sénégal, in: Jahrbuch für afrikanisches Recht, Bd. 3 (1982), S. 137-156. 
Pflege von kranken Arbeitnehmern jährlich einen pauschalen Betrag überweist. Úberall ist auch ein Schutz gegen die Risiken Arbeitsunfall und Berufskrankheit eingeführt. Die meisten Länder kennen auch eine Alters-, Invaliditäts- und Hinterbliebenenversicherung. Das von der französischen Kolonialmacht eingeführte Alterssicherungssystem wurde entweder, wie im Senegal, übernommen und weiter ausgebaut, oder durch andere nationale Alterssicherungssysteme ersetzt. Die bedeutendsten Unterschiede zwischen den einzelnen Systemen betreffen, neben denen technischer Art, die Festlegung der Altersgrenze für die Beziehung einer Rente, die je nach Land zwischen 60 und 50 Jahren liegt.

Der personelle Anwendungsbereich der sozialen Sicherungssysteme erstreckt sich im allgemeinen auf alle lohnabhängigen Arbeitnehmer des formellen Sektors in der Industrie, im Handel, in der Landwirtschaft und im sonstigen Gewerbe. In vielen Ländern können bestimmte, nicht lohnabhängig beschäftigte Personengruppen in den personellen Anwendungsbereich einbezogen werden. Faktisch ausgeschlossen sind jedoch die Arbeitnehmer im traditionellen Agrarbereich und im urbanen informellen Sektor, ${ }^{22}$ so daß im allgemeinen nur eine Minderheit der Gesamtbevölkerung von den Systemen der sozialen Sicherheit profitiert. ${ }^{23}$ Eine Ausnahme bildet Gabun, das den Anwendungsbereich aller Zweige seines Sicherungssystems auf die gesamte Bevölkerung ausgeweitet hat. ${ }^{24}$

\section{Harmonisierung auf der Basis internationaler Normen}

Für die afrikanischen Länder könnte eine Konzeption der Harmonisierung darin bestehen, eine relative Gleichheit hinsichtlich der sozialen Kosten in den verschiedenen Ländern herzustellen. ${ }^{25}$ Dabei würde vor allem das globale Kostenvolumen berücksichtigt, weniger das Leistungsniveau oder die Bedürfnisse der versicherten Arbeitnehmer. Die Frage nach der Finanzierung der Kosten der sozialen Sicherheit wird in den afrikanischen Ländern eingehend untersucht werden müssen, da ihr in Entwicklungsländern wegen der dort vorherrschenden Wirtschafts- und Sozialstruktur noch mehr Bedeutung zukommt als in Industrieländern. Eine Finanzierung über Steuern könnte die so notwendige wirtschaftliche Expansion beeinträchtigen; eine Finanzierung ausschließlich mittels Beiträgen ist dagegen wegen der relativ geringen Zahl von lohnabhängig Beschäftigten sehr problematisch, vor allem in Anbetracht der Tatsache, daß die zahlreichen entsendeten und expatriierten (europäischen) Arbeitnehmer Sozialversicherungsbeiträge im all-

22 Zum Begriff des städtischen informellen Sektors: Sethuraman, S. V. (Hrsg.), The Urban Informal Sector in Developing Countries. Employment, Poverty and Environment, ILO, Genf 1981.

23 Die Ausweitung des Schutzes wird von verschiedener Seite verlangt (s. Cockburn, Ch., Fn. 16); i m Bereich der medizinischen Versorgung durch die Systeme sozialer Sicherheit gibt $Z s c h o c k, D$. K., Analyse générale des problèmes de la fourniture des soins médicaux par la sécurité sociale dans les pays en voie de développement, in: Etudes et recherches no. 18, IVSS, Genf 1982, S. 31, einige Anregungen.

24 S. Gesetz no. 10/52 vom 24. 1. 1983 und internationale Revue für soziale Sicherheit, 1983, S. $287 \mathrm{ff}$.

25 Mouton, $P$., Les problèmes internationaux de la sécurité sociale, in: Encyclopédie juridique de l'Afrique, tome 8, Droit des relations professionnelles, S. 341. 
gemeinen nicht an afrikanische, sondern an europäische Einrichtungen sozialer Sicherheit abgeben.

Die Fünfte Afrikanische Regionalkonferenz der $\mathrm{IAO}^{26}$ hat sich für ein weites Harmonisierungskonzept ausgesprochen. Normen sollten festgesetzt werden, die einen vergleichbaren sozialen Schutz der Arbeitnehmer in den verschiedenen Ländern zum Ziel haben. Die Harmonisierung beschränkt sich also nicht auf allgemeine Maßnahmen auf dem Gebiet der Leistungen, sondern greift auch auf die technischen Garantiemaßnahmen sowie auf die Verwaltungs- und Finanzstrukturen der Systeme über. Dieses Ziel, Harmonisierung durch internationale Konzertation, ist nicht kurzfristig zu erreichen und verlangt eine Abstimmung der nationalen Gesetzgebungen in Anlehnung an von allen Ländern gemeinsam fixierte Normen. Dabei kann sich die Ratifizierung und die Anwendung internationaler Abkommen, insbesondere das IAO-Ubereinkommen Nr. 102 über die Mindestnormen der sozialen Sicherheit, ${ }^{27}$ als die geeignetste Harmonisierungsmaßnahme erweisen. ${ }^{28}$ Schon 1970 kamen die Mitgliedstaaten der Afrikanischen Einheit (OUA) überein, daß einer Harmonisierung dieser Art im Rahmen der allgemeinen Entwicklung eine Priorität eingeräumt werden sollte. ${ }^{29}$ Dieser Standpunkt wurde später bekräftigt. ${ }^{30}$ Würden alle betroffenen Länder ihr System der sozialen Sicherheit den internationalen Normen anpassen, wäre die Harmonisierung zumindest hinsichtlich des Leistungsniveaus erreicht. ${ }^{31}$ Das im Ubereinkommen Nr. 102 vorgegebene Niveau ist in manchen Ländern auch schon erreicht und könnte in anderen durch geringfügige Änderungen der Gesetzgebung erzielt werden. ${ }^{32}$ Das gilt vor allem im Hinblick auf die Möglichkeit einer Teilratifikation des Ubereinkommens Nr. 102. Besonders bedeutsam wäre in den afrikanischen Ländern eine Angleichung der verschiedenen Rechtsordnungen bezüglich der Gewährung von Sach- und Einkommensleistungen für den Fall der Krankheit. Hierbei handelt es sich um einen sehr wichtigen, aber auch besonders kostspieligen Bereich. ${ }^{33}$ Jedoch würde die Ratifizierung des Ubereinkommens Nr. 102 durch alle Staaten nicht genügen, um das Problem der Leistungserbringung, insbesondere hinsichtlich ihrer Voraussetzungen und ihrer Höhe zu lösen. Die verschiedenen Länder müßten sich auf allgemein akzeptierte und gültige Definitionen von Risi-

26 Adidjan 1977 (s. Fn. 16).

27 Vom 28. Juni 1952; s. Zacher, H. F., Internationales und europäisches Sozialrecht, Percha 1976, S. 158 ff.

28 Perrin, G., L'action de l'OIT en faveur de la coordination et de l'harmonisation des législations de sécurité sociale, Revue belge de sécurité sociale, 1969, S. 1115.

Euzéby, $A$., Développement de la sécurité sociale e t nouvel ordre économique international. Etudes e t travaux économiques 1, Cahiers de la Faculté des sciences économiques de Grenoble, S. 101.

29 7. OUA-Konferenz.

Perrin, G., Les fondements du droit international de la sécurité sociale, Droit social 1974, S. 479.

30 4. Afrikanische Regionalkonferenz der ILO, Nairobi 1973. 5. Afrikanische Regionalkonferenz der ILO, Abidjan 1977.

31 Ayissi Mvodo, V./Le Faou, R., L'Influence des normes internationales du travail sur le législation camérounaise, Revue internationale du travail, avril/septembre 1973.

32 Improvement and Harmonisation of Social Security (Fn. 16), S. 61.

33 AISS (IVSS), Financement et contrôle du coût des services de soins médicaux, in: Les soins médicaux fournis par la sécurite sociale dans les pays en développement (Fn. 23), S. $115 \mathrm{ff}$. 
ken, wie z. B. der Risiken Berufskrankheit oder Invalidität, einigen. Dabei käme die internationale Konzertation zum Zuge, durch welche Abweichungen zwischen den einzelnen Rechtsordnungen ausgeschaltet würden. Denn das Úbereinkommen Nr. 102 läßt den Mitgliedstaaten verschiedene Wahlmöglichkeiten offen. Das kann vor allem anhand der im Úbereinkommen vorgesehenen Wahlmöglichkeit bezüglich des geschützten Personenkreises deutlich gemacht werden. Die Forderungen des Ubereinkommens sind erfüllt, wenn in dem betreffenden Land ein bestimmter Prozentsatz aller Arbeitnehmer, aller Erwerbstätigen oder aller Einwohner unter den Anwendungsbereich des Sicherungssystems fallen. Um sich auf eine dieser drei Möglichkeiten zu einigen, müßten die Länder also einer internationalen Solidarität Ausdruck verleihen, die ihren nationalen Interessen ggf. zuwiderlaufen könnte, je nach Art der Sozialstruktur des einzelnen Landes. ${ }^{34}$ Das Problem ist also zugleich politischer und wirtschaftlicher Natur.

Die internationale Arbeitsorganisation wirkt nicht nur mittels ihrer Abkommen auf die Harmonisierung der verschiedenen afrikanischen Systeme ein, sondern auch mittels der von ihr durchgeführten internationalen technischen Zusammenarbeit. Eines der eloquentesten Beispiele dafür ist die in den 1960er Jahren realisierte Harmonisierung der Alterssicherungsysteme in einigen francophonen Ländern. Dabei wurde das von der Institution de Prevoyance Retraites de l'Afrique Occidentale Française (Alterssicherungssystem, IPRAO) ${ }^{35}$ nach der Unabhängigkeit von den einzelnen Ländern übernommene komplizierte Punktesysteme ${ }^{36}$ durch ein einfacheres und den afrikanischen Gegebenheiten besser angepaßtes System ersetzt. ${ }^{37}$

Gerade weil eine Harmonisierung nur langfristig erreicht werden kann, müssen die betroffenen Staaten noch andere Maßnahmen ergreifen, die geeignet sind, die Nichtübereinstimmungen der nationalen Gesetzgebung zu überbrücken. Dabei greifen die Koordinierungsmaßnahmen ein.

34 Folgende Zahlen können einen Uberblick geben: In den Ländern mit niedrigem Einkommen (Benin, Burundi, Guinea, Madagaskar, Mali, Mauretanien, Niger, Obervolta, Ruanda, Somalia, Sudan, Togo, Tchad, Zaire, Zentralafrikanische Republik), sind $71 \%$ der Beschäftigten in der Landwirtschaft tätig, $14 \%$ in der Industrie und $15 \%$ im Dienstleistungsbereich.

Für die Länder mit mittlerem Einkommen (Elfenbeinküste, Gabun (ölexportierendes Land) Kamerun, Senegal) gelten folgende Zahlen: $43 \%, 23 \%, 34 \%$.

Quelle: Worldbank, Accelerated Development in Subsaharan Africa, 1981, S. 178.

35 Dazu: Mouton, $P$., L'étendue des systèmes africains de sécurité sociale, in: Encyclopédie juridique de l'Afrique (Fn. 25), S. 315.

36 Jeder Versicherte erwirbt jedes Jahr eine bestimmte, von der Beitragshöhe abhängende Anzahl von Punkten. Die Rentenhöhe entspricht der Gesamtzahl der Punkte, die mit dem Wert eines Punktes multipliziert wird.

37 Ausnahme: Senegal, s. Kaufmann, O., Le droit de la sécurité sociale au Sénégal, (Fn. 21), sowie: Grundzüge des Rechts der sozialen Sicherheit im Senegal, Zeitschrift für vergleichende Rechtswissenschaft, 1983, S. $248 \mathrm{ff}$. 


\section{Koordinierungsmaßnahmen im internationalen Rahmen: multilaterale Abkommen}

Die Koordinierungsmaßnahmen, deren Zweck es ist, eine Diskriminierung der Wanderarbeiter zu verhindern, erfüllen verschiedene Funktionen und gewährleisten die Gleichstellung und Gleichbehandlung aller Arbeitnehmer, die Anwendung einer einzigen Gesetzgebung auf dasselbe Versicherungsverhältnis, die Wahrung erworbener und im Erwerb befindlicher Rechte und Anwartschaften sowie die Möglichkeit der Leistungserbringung auch im Ausland. ${ }^{38}$ Wie im Bereich der Harmonisierung, können auch hier internationale Úbereinkommen der IAO bis zu einem gewissen Grad die Probleme lösen. Dabei kommen insbesondere das Ubereinkommen Nr. 19 über die Gleichbehandlung bei Arbeitsunfällen ${ }^{39}$ und das Ubereinkommen Nr. 118 über die Gleichbehandlung von Inländern und Ausländern in der sozialen Sicherheit ${ }^{40}$ in Betracht. Auch bilaterale Abkommen können eine Rolle spielen. Aber fast alle bilateralen Abkommen auf dem Gebiet der sozialen Sicherheit wurden zwischen afrikanischen Ländern und ihrer ehemaligen Kolonialmacht abgeschlossen und haben infolgedessen eine andere Zielsetzung, da sie in erster Linie auf die Wanderbewegungen zwischen Afrika und Europa abgestimmt sind. ${ }^{41}$ Die größte Bedeutung für die Koordinierung haben regionale multilaterale Abkommen, insbesondere das Abkommen über die soziale Sicherheit der Wanderarbeitnehmer der Organisation Commune Africaine et Malgache (OCAM, Gemeinsame Afrikanische-Mauritische-Malgesische Organisation) und das Abkommen der Communauté Economique des Pays des Grands Lacs (CEPGL, Wirtschaftsgemeinschaft der Länder der Großen Seen).

Das OCAM-Abkommen, unter Mitarbeit des IAA entstanden, baute auf den Vorarbeiten der Organisation der Anliegerstaaten des Senegalflusses auf und trat 1971 in Kraft. ${ }^{42}$ Trotz der relativ kleinen Zahl von Mitgliedstaaten der OCAM muß ihr Abkommen als das wichtigste angesehen werden. ${ }^{43}$

38 Dazu: Diallo, M., Les problèmes de la sécurité sociale des travailleurs migrants en Afrique, IVSS, Documents de sécurité sociale, série africaine, no. 4, 1981, S. $41 \mathrm{ff}$.

39 Vom 5. Juni 1925 s. Zacher (Fn. 27), S. 255 ff. Verbindlich für: Burundi, Elfenbeinküste, Gabun, Kamerun, Madagaskar, Mali, Mauretanien, Obervolta, Ruanda, Senegal, Zaire, Zentralafrikanische Republik.

40 Vom 28. Juni 1962 s. Zacher (Fn. 27), S. 188 ff. Verbindlich für: Guinea, Madagaskar, Mauretanien, Zaire, Zentralafrikanische Republik.

41 Senegal hat ein Abkommen mit Mali und Mauretanien abgeschlossen. Zwischen Senegal und Gabun besteht ein Vereinbarungsprotokoll. Folgende francophone Länder Schwarzafrikas haben ein Abkommen mit Frankreich geschlossen: Benin, Gabun, Madagaskar, Mali, Mauretanien, Niger, Obervolta, Senegal, Togo. Zwischen Belgien und Zaire besteht ein Abkommen, dessen personeller Anwendungsbereich auf die Seefahrer beschränkt ist.

42 S. Legislative Series 1971 - Int. 1, ILO, Genf, und Journal Official du Togo vom 1. 10. 1971.

Zur Entstehung: Sawadago, J. M., La convention générale de sécurité sociale des travailleurs migrants OCAM, in: BIT, Rapport des seminaires régionales africains, BIT/Norvège sur l'application de la convention générale de sécurité sociale (OCAM), Lomé 27. Nov./9. Dez. 1978, ILO/TF/AFR/R 29, S. 16 ff. und: Voirin, M., Social Security for Migrant Workers in Africa, International Labour Review 1983, S. $333 \mathrm{ff}$.

43 Die OCAM zählte bis zu 15 Mitgliedstaaten. Die jetzigen Mitgliedstaaten sind: Benin, Elfenbeinküste, Mauritius, Niger, Obervolta, Ruanda, Senegal, Togo, Zentralafrikanische Republik; Kongo und Tchad gehören der OCAM nicht mehr an, scheinen aber das Abkommen weiterhin als verbindlich anzusehen. 
Der Geltungsbereich des OCAM-Abkommens erstreckt sich auf alle Zweige der bestehenden nationalen Gesetzgebungen, insbesondere auf die Bereiche Alter, Invalidität, Hinterbliebenensicherung, Arbeitsunfall und Berufskrankheiten, Familienleistungen, Mutterschaftsleistungen, sowie Leistungen im Krankheitsfall. ${ }^{44}$ Alle Systeme der sozialen Sicherheit sind in den Geltungsbereich einbezogen, unabhängig von ihrer rechtlichen oder technischen Natur, also auch die, bei denen die Leistungserbringung dem Arbeitgeber übertragen worden ist. Das Abkommen ist auf unbestimmte Zeit abgeschlossen und gilt auch für zukünftige Systeme sozialer Sicherheit eines jeden Unterzeichnerstaates. Jedoch kann jeder Staat das Abkommen kündigen.

Der personelle Geltungsbereich erstreckt sich auf alle versicherten Arbeitnehmer, die die Nationalität eines Vertragsstaates haben und der Gesetzgebung eines oder mehrerer Vertragsstaaten unterworfen sind oder waren. Wer als Familienangehöriger leistungsberechtigt ist, ergibt sich aus der zuständigen nationalen Gesetzgebung. Ausgeschlossen sind lediglich die öffentlich Bediensteten, deren Schutzsysteme nicht in den Anwendungsbereich des Abkommens fallen.

Die oben genannten Prinzipien, deren Verwirklichung die Koordinierung dient, also Gleichbehandlung, Einheit der anzuwendenden Gesetzgebung, Wahrung der Rechte, Leistungsexport, werden vom Abkommen und seinen Zusatztexten angestrebt. Ausdrücklich wird das Prinzip der Gleichbehandlung der ausländischen und inländischen Arbeitnehmer erwähnt. Das auf einen Leistungsfall anzuwendende Recht ist das Recht des Beschäftigungslandes. Diese Bestimmung ist unabhängig vom Wohnsitz des Beschäftigten oder vom Sitz des Unternehmens. Von diesen Bestimmungen kann für den Fall der Entsendung abgewichen werden, wenn die Entscheidungsdauer sechs Monate nicht überschreitet, wobei eine Verlängerung um die gleiche Dauer zulässig ist. Eine weitergehende Verlängerung, bis zur Beendigung einer bestimmten Arbeit, ist möglich unter der Voraussetzung, daß die zuständigen nationalen Stellen diesbezüglich eine Vereinbarung treffen. Abweichende, aber präzise Bestimmungen enthält das Abkommen für den Fall einer Beschäftigung in mehreren Ländern, sowie für die Schiffahrt. Um mögliche Konflikte zwischen zwei oder mehreren Systemen zu verhindern, sieht das Abkommen mehrere Lösungen vor: eine freiwillige Weiterversicherung steht immer hinter einer Pflichtversicherung zurück. Bestehen mehrere freiwillige Weiterversicherungsverhältnisse, so ist entweder das Versicherungsverhältnis des Aufenthaltlandes anwendbar oder dasjenige der Rechtsordnung, die zuletzt auf das Beschäftigungsverhältnis anwendbar war. Eine Wahlmöglichkeit ist ausgeschlossen, auch wenn es vom Günstigkeitsprinzip her geboten schiene.

Auch hinsichtlich der Leistungserbringung enthält das Abkommen präzise Vorschriften. Geldleistungen, insbesondere Mutterschafts- und Familienleistungen, werden von den leistungspflichtigen Institutionen in voller Höhe unter Anrechnung aller Beschäftigungszeiten in den verschiedenen Ländern gezahlt. Invaliditäts-, Alters- und Hinterbliebenenleistungen werden unter Anrechnung aller Versicherungszeiten und unter Zugrundele- 
gung einer pro-rata-Berechnung gewährt. Für das Risiko Arbeitsunfall sieht das Abkommen für die Gewährung von Sachleistungen vor, sie nach den Bestimmungen des Aufenthaltslandes zu erbringen, wobei aber die Leistungsberechtigung nach den Bestimmungen festgestellt wird, denen die leistungspflichtige Institution unterliegt. Geldleistungen werden auch im Ausland von der leistungspflichtigen Institution gemäß den Bestimmungen, denen sie unterliegen, gezahlt.

Obwohl das Abkommen bereits vor 13 Jahren in Kraft getreten ist, scheint es erst jetzt nach und nach wirklich zur Anwendung zu kommen..$^{45}$ Das liegt wohl auch darin begründet, daß die im Abkommen vorgesehenen Kooperationsmaßnahmen zwischen den verschiedenen nationalen Systemen, durch welche eine Koordinierung erst praktisch relevant wird, erst jetzt zum Zuge kommen. Es handelt sich insbesondere um Maßnahmen auf dem Gebiet des Informationsaustauschs, der Verwaltungspraxis und des Verfahrensrechts. Ein anderer Grund für die Nichtanwendung des Abkommens dürfte der hohe technische Standard des Abkommens sein, der sich mit den vorhandenen Kapazitäten einiger afrikanischer Systeme sozialer Sicherheit nicht ohne weiteres in Einklang bringen läßt. ${ }^{46}$ Es soll aber nicht vergessen werden, daß das OCAM-Abkommen direkte Auswirkungen auf andere internationale Instrumente gezeigt hat. ${ }^{47}$

Ein weiteres wichtiges Abkommen wurde 1978 von der Wirtschaftsgemeinschaft der Länder der Großen Seen (CEPGL) unterzeichnet und trat 1980 in Kraft. ${ }^{48}$ Das CEPGLAbkommen hat größtenteils die Bestimmungen des OCAM-Abkommens übernommen. Aber da die einschlägigen Gesetzgebungen der drei Mitgliedstaaten, Burundi, Ruanda und Zaire, die früher der belgischen Kolonialmacht unterstanden, nur in einem sehr geringen Umfang Mutterschafts- und Familienleistungen vorsehen, sind diese beiden Bereiche der sozialen Sicherheit nicht Bestandteil des Abkommens. Wie das OCAMAbkommen, ist auch das CEPGL-Abkommen noch nicht voll zur Anwendung gelangt. Zwei weitere afrikanische Organisationen bereiten mit Hilfe des IAA Abkommen über die soziale Sicherheit vor. ${ }^{49}$ Es handelt sich um die westafrikanische Wirtschaftsgemeinschaft (CEAO, Communauté Economique de l'Afrique Occidentale) ${ }^{50}$ und die Zentralafrikanische Zoll- und Wirtschaftsunion (UDEAC, Union Douanière et Economique de l'Afrique Centrale), ${ }^{51}$ die schon 1977 die Durchführung von Koordinierungsmaßnahmen beschlossen hat. Jedoch sind diese Projekte sehr ungewiß. ${ }^{52}$

45 Mouton, P., Les problèmes internationaux de la sécurité sociale, in: Encyclopédie (Fn. 25), S. 354.

46 Dem Abkommen diente als Modell das Europäische Abkommen über soziale Sicherheit. S. Perrin, G., Un nouvel instrument multilatéral pour la protection des travailleurs migrants, in: Revue belge de sécurité sociale, no. 1, 1973.

47 Insbes. auf die Abkommen mit Frankreich, S. Mouton, P., Les problèmes internationaux de sécurité sociale (Fn. 25), S. 352.

48 Recueil des textes et accords constitutifs et des conventions de la CEPGL, Gisenyi, 1979. Und: BIT, Rapport du seminaire régional africain BIT/Belgique sur l'application de la convention générale de sécurité sociale (CEPGL), Gisenyi (Rwanda), 5 au 17 mai 1980, ILO/TF/AFR/R 31, Genève 1981.

49 Voirin, M., Social Security for Migrant Workers in Africa (Fn. 42), S. 335.

50 Die Mitgliedstaaten sind: Elfenbeinküste, Mali, Mauretanien, Niger, Obervolta, Senegal.

51 Die Mitgliedstaaten sind: Gabun, Kongo, Zentralafrikanische Republik.

52 Décision no. 6/77 UDEAC - 70 du 21. 12. 77 portant directives pour la coordination des législations de sécurité sociale. Abgedr. in: J.O. de la République Gabonaise du 15 février 1978, p. 201. 


\section{Schlußbetrachtungen}

Zweifellos wurden auf dem Gebiet der sozialen Sicherheit sowohl im nationalen, als auch im internationalen Rahmen bedeutende Fortschritte erzielt, die gerade im internationalen Rahmen in Zukunft immer mehr Früchte tragen werden. Jedoch darf nicht übersehen werden, daß die unterschiedliche nationale Entwicklung die internationale Zusammenarbeit bremsen kann. Das trifft schon für Länder zu, die aufgrund geschichtlicher Gegebenheiten ähnliche Strukturen aufweisen, wird aber noch stärker zum Vorschein kommen, wenn systemüberwindende Koordinierungsmaßnahmen praktisch angegangen werden sollen. Das wäre der Fall, wenn die Mitgliedstaaten der Wirtschaftsgemeinschaft der Westafrikanischen Staaten (ECOWAS) tatsächlich ein Abkommen über soziale Sicherheit ausarbeiten. In diesem Fall wären nicht nur francophone, sondern auch anglo- und lusophone Staaten beteiligt, deren Systeme sehr große Differenzen aufweisen. ${ }^{53}$

Zum jetzigen Zeitpunkt geht es aber primär darum, die vorhandenen Harmonisierungsund Koordinierungsinstrumente praktisch nutzbar zu machen. Es kann festgestellt werden, daß eine Harmonisierung zum derzeitigen Zeitpunkt - und höchst wahrscheinlich auch auf lange Sicht - für die afrikanischen Länder nur darin bestehen kann, ihre Sozialgesetzgebungen dem Niveau internationaler Instrumente anzugleichen. Eine bis zur Integration weitergehende Harmonisierung ist wegen der zu großen Disparitäten in den einzelnen Ländern nicht durchführbar. Aber sie ist auch nicht notwendig, da Koordinierungsinstrumente verfügbar sind. Das OCAM - wie auch das CEPGL-Abkommen - sind funktionsfähig und können durch ihre tatsächliche Anwendung den sozialen Schutz der Wanderarbeitnehmer garantieren. Konflikte, die sich durch die Ratifizierung beider Abkommen durch ein Land (Ruanda) ergeben, müssen eben bis zum Zeitpunkt einer geographisch weitergefaßten Koordinierung, in Kauf genommen werden. Unverzichtbar ist es aber, der in den Abkommen vorgesehenen zwischenstaatlichen Kooperation Leben zu geben, und sie immer weiter auszubauen. Dabei darf natürlich nicht außer Acht gelassen werden, daß schon innerhalb der einzelnen nationalen Rechtsordnungen in vielen Bereichen schwerwiegende Mängel auftreten, ${ }^{54}$ die es auszuschalten gilt.

Im Interesse der Bevölkerung der einzelnen Staaten sollte auch die Ausweitung der nationalen Sicherungssysteme in Angriff genommen werden, allein schon deshalb, um einer "umgekehrten Diskriminierung", nämlich der innerhalb einer Rechtsordnung verbleibender Arbeitnehmer gegenüber den Wanderarbeitnehmern, vorzubeugen.

Zur ECOWAS, s. Onvuka, R. I., Development and Integration in Westafrica (ECOWAS), University of Ife Press, 1982.

54 Improvement and Harmonisation (Fn. 16). 


\section{International Aspects of Social Security in French-Speaking Countries of the Sahara}

\section{By Otto Kaufmann}

The scanty social security legislation introduced during the colonial period by French and Belgian colonial administrations was applied in all colonial territories. Therefore, problems concerning social security of migrant workers did not exist.

After independence, the new African countries each adopted national social security legislation. Despite the common French or Belgian influence, differences appeared between the different national social security systems. The reasons for these differences are various, but their consequence is the necessity to take measures in favour of African migrant workers.

Indeed, in the absence of coordination or harmonization of national social security legislations, migrant workers may be disadvantaged. Measures in the field of harmonization of social security legislation may consist in ratifying ILO conventions, especially the convention no. 102. But harmonization, for several reasons, has to be completed by coordination measures. The African countries concerned could apply the ILO's coordination principles and standards. But one can observe a predominance of multilateral solutions within African subregional organizations. The existing multilateral instruments are the social security convention of the Common African and Mauritian Organization (OCAM) and the conventions of the Economic Community of the Countries of the Great Lakes (CEPGL). The two conventions comprise the principle of national treatment, rules for the determination of the applicable law, the maintenance of acquired rights and the maintenance of rights in course of acquisition. There are still many obstacles to overcome, but this manner of coordination seems to be the best to guarantee the social rights of the migrants. That is why other African organizations are also engaged in negotiations about social security.

\section{Legislative Comprehensiveness - An Assessment of Two Tanzanian Provisions}

\section{By Zebron Steven Gondwe}

It is now probably settled that law is an obligatory rule of conduct, it enjoins or prohibits certain action in a community. However, full utility of law is possible where it comprehensively provides for the situation sought to be governed. The article, citing two Tanzanian legal provisions as illustrations, shows how legal comprehensiveness may be impaired. First the author identifies the inadequacy of a legal provision regarding the situation(s) intended to be covered thereby. It is shown that the provision is not exhaustive in that regard. A solution is recommended for examination and possible adoption. In contrast the other provision examined is substantially adequate but its literary presentation 